\title{
Pulmonary Artery Aneurysms Revealing Behçet's Disease
}

\author{
Hajar EL Mortaji ${ }^{*}$, Hanane EL Hammaoui, Badr Boutakioute, Mariem Ouali Idrissi, Najat Cherif Idrissi Ganouni
}

Département de Radiologie, Hôpital Arrazi, CHU MED VI, Marrakech, Marocoo

DOI: $10.36347 /$ sjmcr.2020.v08i10.011

| Received: 27.01.2020 | Accepted: 03.02.2020 | Published: 19.10.2020

*Corresponding author: El Mortaji Hajar

\section{Abstract}

\section{Original Research Article}

Pulmonary arterial aneurysm is defined by a dilation of the pulmonary artery. It should not be confused with a false aneurysm; Witch is due to a breach in the vessel wall. Known since the 18th century when syphilis and tuberculosis were endemic, aneurysms of the pulmonary artery became rare with the advent of antibiotics. In Behçet's disease, arterial involvement is rare and has a poor prognosis. We report a case of bilateral aneurysms involving the lobar and segmental pulmonary arteries during Behçet's disease in a 45-year-old patient who presented with hemoptysis. The chest X-ray showed bilateral round pulmonary opacities. The thoracic CT scan showed multiple bilateral aneurysms of the lobar and segmental pulmonary arteries. The evolution was spectacular under medical treatment. The peculiarity of this case lies in the rarity of arterial involvement during Behçet's disease in general and in the rarity of the segmental arterial involvement in particular; as well as in the good evolution under medical treatment even with the presence of hemoptysis.

Keywords: Pulmonary artery aneurysm, Behçet's disease, round opacity, images.

Copyright $\left({ }_{0} 2020\right.$ The Author(s): This is an open-access article distributed under the terms of the Creative Commons Attribution 4.0 International License (CC BY-NC 4.0) which permits unrestricted use, distribution, and reproduction in any medium for non-commercial use provided the original author and source are credited.

\section{INTRODUCTION}

Behçet's disease is a systemic vasculitis which mainly affects young people. Arterial involvement during Behçet's disease is rare and may reveal the disease $[1,2]$. It has a poor prognosis [3, 4].

\section{CaSe RePORT}

A 45-year-old male patient, not known to smoke, with a history of recurrent oral aphthosis, presented to our department with episodes of mild hemoptysis. He has a normal cardiovascular examination. The general examination found a genital ulceration in the scrotum. The chest X-ray revealed two round and well defined opacities, with a right para hilar and a left para cardiac projections (Figure 1). The computed tomography showed multiple bilateral basal lesions, with a round shape and polycyclic contours, of liquid density on spontaneous contrast (figure 2a) simultaneously and similary enhanced compared to the pulmonary artery trunk (Figures $2 \mathrm{~b}, 2 \mathrm{c}$ ). The largest of these aneurysms was located in the arterial branch of the right latero-basal segment and measured $22 \times 17$ $\mathrm{mm}$. The rest of the lung parenchyma was normal. The diagnosis of Behçet's disease with pulmonary arterial aneurysms was evoked. The patient received 12 boluses of cyclophosphamides. During his evolution, the patient had no longer hemoptysis. In the scanographic control carried out 2 years later, we noted the persistence of the largest aneurysm currently measuring $9.3 \times 8.3 \mathrm{~mm}$ with the total regression of the other aneurysmal dilations (Figure 3).

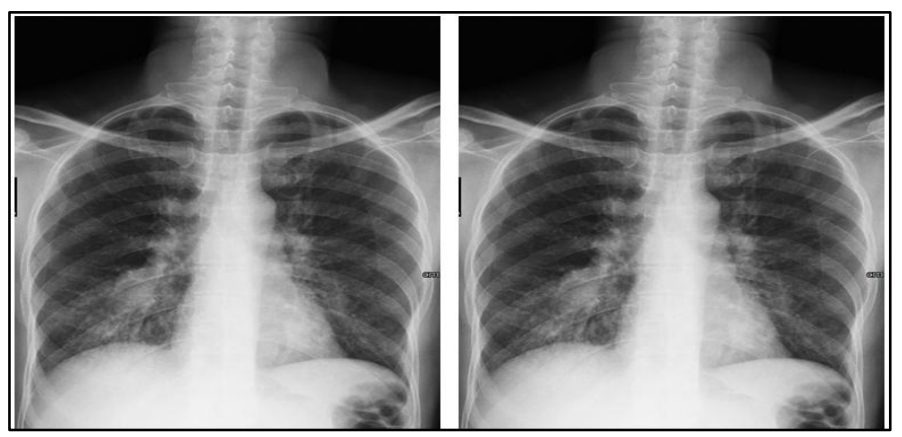

Fig-1: Chest X-ray showing two round and well defined opacities, with right para hilar and left para cardiac projections 


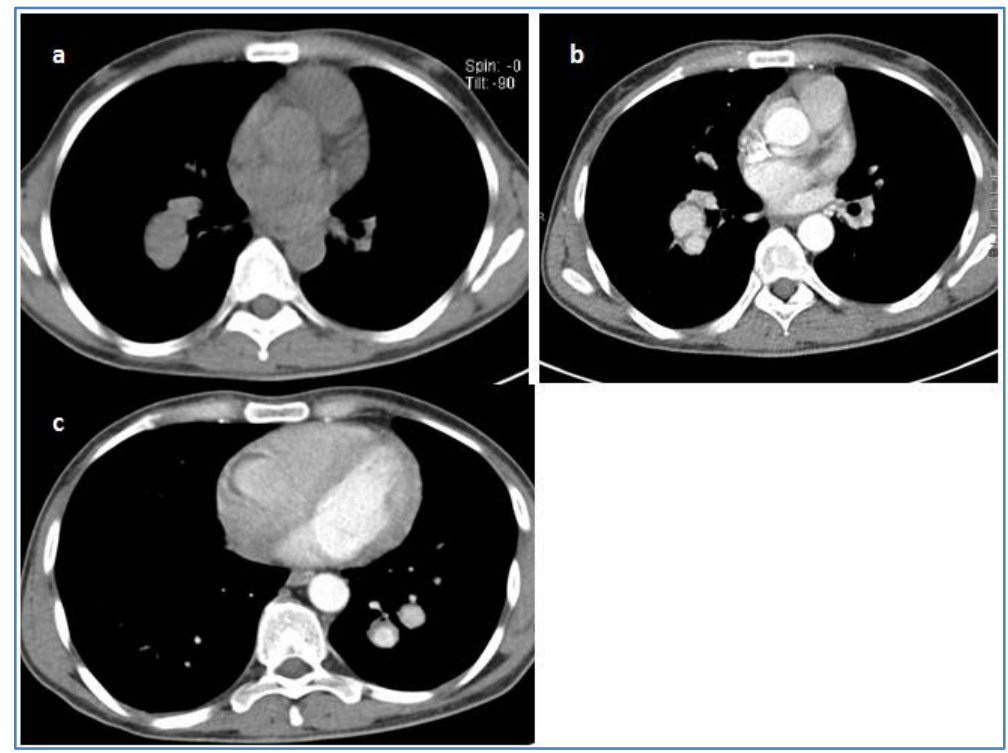

Fig-2: CT scan axial images showing multiple and bilateral basal lesions, with a round shape and polycyclic contours, of liquid density on spontaneous contrast (figure 2a) simultaneously and similary enhanced compared to the pulmonary artery trunk (Figures 2b, 2c)

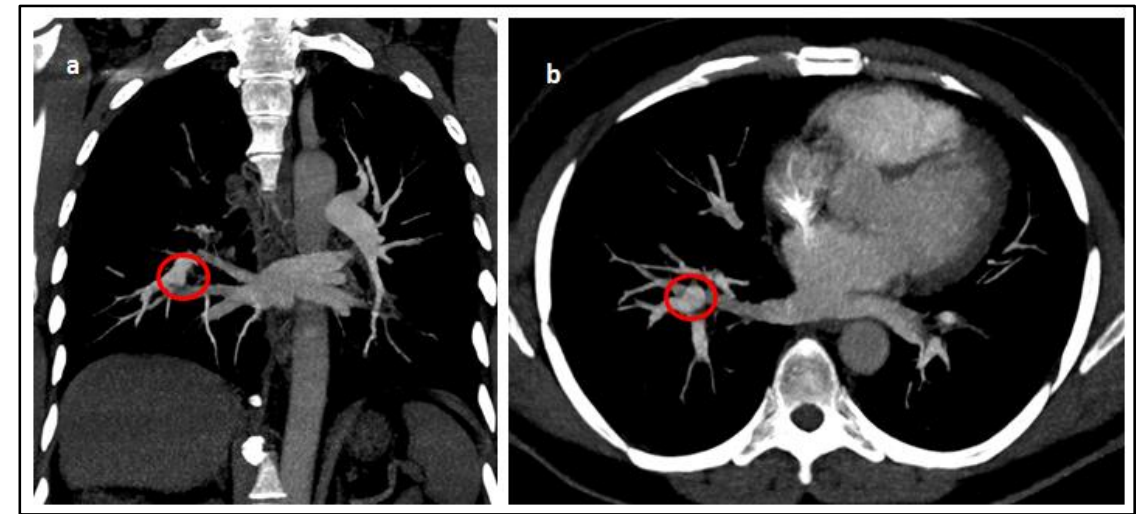

Fig-3: CT scan images with coronal (a) and axial (b) views showing a partial regression of the aneurysm of the arterial branch of the right latero-basal segment (cercle) with a total regression of the other aneurysmal dilations

\section{DISCUSSION}

Behçet's disease is a relapsing and remitting chronic inflammatory disease of an unknown origin [5]. It affects young subjects, generally between the age of 20 and 30 years old with a male predominance [1].

Classicaly described as a triad including oral and genital aphtosis associated with uveitis. The systemic manifestations can be variable including skin, joint, vascular, pulmonary, cerebral, digestive and urogenital lesions. The commun anatomopathological substratum of all those lesions is vasculitis [5].

Thoracic involvement is rare (1-8\%). It usually includes thrombosis of the superior vena cava, pulmonary arterial aneurysms and rarely pulmonary infarction and alveolar hemorrhage [4].

The mortality in patients with Behçet's disease is 3 to $4 \%$ apart from a pulmonary involvement; it can be up to $50 \%$ when pulmonary arterial aneurysms are associated [6].
Aneurysms of the pulmonary artery during Behçet's disease are very rare. They can reveal the disease, as it is the case of our patient. They usually occure on both sides in the lobar pulmonary arteries and the arterial pulmonary trunk, or more rarely on the segmental pulmonary arteries [7].

Standard radiographics show an enlargement of the mediastinum related to proximal pulmonary arterial aneurysms and round opacities when the aneurysms occure in the distal arterial branches [8]. Due to the non-specific nature of these signs, the range of differential diagnoses is wide: venous thromboembolic disease, pulmonary arterial hypertension, mediastinal and hilar nodes, bronchopulmonary cancer, metastasis, and even hydatid disease, given our epidemiological context [9].

CT scan is the gold standard to illustrate the wide spectrum of anomalies encountered in Behçet's disease [5]. Pulmonary aneurysms are recognised by the enlargement of the affected artery with regular or irregular border, fusiform or saccular shape. Wall 
thrombosis is common. In case of complete aneurysmal thrombosis, the diagnosis with a thromboembolic venous disease is very difficult. The coexistence of deep vein thrombosis, other pulmonary arterial thrombosis without aneurysm and pulmonary infarction are all elements that plead to the diagnosis [10].

The characteristics of pulmonary arterial aneurysms have been specified by Tunaci et al. [10] in their series of 13 patients. $24 \%$ occure on the trunk of the pulmonary artery, $54 \%$ on a lobar artery and $22 \%$ on a segmental artery. They are more frequent on the right site $(59 \%)$, predominant in the lower lobes and are more often multiple and bilateral.

These radiological findings are similar to those of mycotic aneurysms. Apart from the different clinical presentation, some radiological signs can point to Behçet's disease [9] such as the speed of progression, the asymmetrical development of aneurysms of different ages, associated homolateral pleuropulmonary anomalies and thromboses of the superior vena cava.

MRI has the advantage to detect signs of aneurysmal wall rupture and to evaluate how old the thrombus is [11]. Coupled with the CT scan, MRI appears to be an effective tool for the etiological diagnosis of hemoptysis thanks to its ability to illustrate intravascular, intra mural and extramural anomalies [12].

Therapeutic management of Behçet's disease is difficult, especially when hemoptysis and aneurysms of the pulmonary arteries are associated [13].

Surgical treatment of aneurysms consists of excising them [7]. It should be noted that surgery for pulmonary aneurysms is particularly dangerous. The only successful reported cases treated surgically were limited to lobectomies removing both of the aneurysm and the concerned lobe [14]. Some cases of regression of the aneurysms have been obtained with medical treatments (corticosteroids, cyclophosphamides or thalidomide). Embolization is a minimally invasive therapy that remains the most promising nowadays [7].

However, the occurrence of hemoptysis comes to disrupt the therapeutic management. It indicates a pulmonary or bronchial cracking of the aneurysmal wall. It is also responsible of a higher rate of mortality that reaches $50 \%$ within an average survival rate of 9.5 months as soon as the hemoptysis appears [6]. Hence the importance of early diagnosis and considering a warning sign: hemoptysis!

\section{REFERENCES}

1. Tazi-Mezalek Z, Ammouri W, Maamar M. Les atteintes vascu-laires au cours de la maladie de Behc et. Rev Med Interne. 2009;30:232-7

2. Hassikou H, Bono W, Bahiri R, Abir S, Benomar M, HajjajHassouni N. Angiobehc et. A propos de deux cas. Rev Rhum. 2002;69:759-62

3. Uzun O, Akpolat T, Erkan L. Pulmonary vasculitis in Behc, etdisease. A cumulative analysis. Chest. 2005; 127:2243-53

4. Hamuryudan V, Er T, Seyahi E, Akman C, Tüzün $\mathrm{H}$, Fresko I. Pulmonaryarteryaneurysms in Behc et syndrome. Am J Med. 2004;117:867-70

5. Erkan F, Gul A, Tasali E. Pulmonary manifestations of Behçet's disease. Thorax. 2001; 56: $572-578$.

6. Hamuryudan V, Yurdakul S, Moral F. Pulmonary arterial aneurysms in Behçet'ssyndrome : a report of 24 cases. Br J Rheumatol. 1994; 33: 48- 51.

7. Bachmeyer C, Khalil A, Bouvard E, Parrot A. Une he' moptysieaucours d'une maladie de Behc et. Rev Med Interne. 2007; 28:784-6.

8. Ahn JM, Im JG, Ryoo JW. Thoracic manifestations of Behçetsyndrome : radiographic and CT findings in nine patients. Radiology. 1995; 194: 199- 203.

9. Hassine E, Bousnina S, Marniche K, et al. Anévrismes des artères pulmonaires au cours de la maladie de Behçet. Contribution de l'imagerie. Cinq observations. Ann Med Interne 2002; 153: 147- 152.

10. Tunaci A, Berkmen YM, Gokmen E. Thoracic involvement in Behçet'sdisease: pathologic, clinical, and imaging features. Am J Roentgenol. 1995; 164: $51-56$.

11. Akpolat T, Danaci M, Belet U. MR imaging and MR angiography in vascular Behçet's disease. MagnReson Imaging. 2000; 18: 1089- 1096.

12. Greene RM, Saleh A, Taylor AK. Non- invasive assessment of bleeding pulmonary artery aneurysms due to Behçet disease. Eur Radiol. 1998; 8: 359- 363.

13. Wechsler B, Le Thi Huong DU, Godeau P. Le traitement de la maladie de Behçet. Sem Hôp Paris. 1986; 62: 1341- 1344.

14. Papa M, De Cobelli F, Baldissera E. Takayasu arteritis: intravascular contrast medium for MR angiography in the evaluation of disease activity. Am J Roentgenol. 2012; 198: 279- 284. 